\title{
CANADIAN JOURNAL OF
}

\section{Disability Studies}

\section{Canadian Journal of Disability Studies}

Published by the Canadian Disability Studies Association

Association Canadienne des Études sur l'Incapacité

Hosted by The University of Waterloo

www.cjds.uwaterloo.ca

Ce document a été rendu accessible et conforme aux normes PDF/UA par Accessibil-IT Inc. Pour plus d'informations, rendez-vous à http://www.accessibilit.com 


\title{
Mobilisation Des Ressources De Soutien Formel Des Parents Immigrants D'un Enfant En Situation De Handicap
}

\author{
Carine Bétrisey, Ph.D. \\ Doctorat sur mesure en Interculturalité et Handicap \\ Université Laval, Québec (QC), Canada \\ carine.betrisey.1@ulaval.ca
}

\section{Résumé}

Plus vulnérables que d'autres personnes, les parents immigrants d'un enfant en situation de handicap possèdent des ressources altérées par leur parcours migratoire. Souvent, ils mobilisent davantage de soutien formel afin de faire face aux événements déstabilisants du quotidien. La présente étude vise à explorer cette situation avec huit entrevues semidirigées menées auprès de parents immigrants dans la province de Québec. L'analyse exploratoire des propos des participants met en évidence sept principales sources de stress expliquant l'utilisation de stratégies d'aide formelle. Des besoins non comblés sont également ressortis. Un modèle explicatif original illustrant ce contexte parental particulier est proposé.

\section{Mots clés}

immigration, situation de handicap, famille, soutien.

\begin{abstract}
The immigrant parents of children with disabilities are more vulnerable than others and they own reduced resources by the migration process. They often mobilize more formal support, in order to cope with everyday life destabilizing events. This study aims to explore this situation with eight semi-structured interviews conducted with immigrant parents settled in the province of Quebec. The exploratory analysis of what was said by the participants highlights seven significant stress factors explaining the use of formal support strategies. Unmet needs were also noted. An original explanatory model is proposed to illustrate this particular parental context.
\end{abstract}

\section{Keywords}

immigration; disability; family; support. 


\title{
Mobilisation Des Ressources De Soutien Formel Des Parents Immigrants D'un Enfant En Situation De Handicap
}

\author{
Carine Bétrisey, Ph.D. \\ Doctorat sur mesure en Interculturalité et Handicap \\ Université Laval, Québec (QC), Canada \\ carine.betrisey.1@ulaval.ca
}

\section{Introduction}

Quitter son pays d'origine de manière volontaire ou non pour s'installer dans un autre amène des défis considérables. Lorsque s'ajoute à ce parcours migratoire la naissance d'un enfant en situation de handicap (liée à une incapacité motrice, langagière, sensorielle, cognitive ou comportementale), les épreuves se multiplient et s'intensifient au fur et à mesure que 1'enfant grandit (Hodgetts, Zwaigenbaum et Nicholas, 2015; Khanlou, Haque, Sheehan et Jones, 2014; Povenmire-Kirk, Lindstrom et Bullis, 2010). Ces parents immigrants se trouvent généralement confrontés à une accumulation d'événements de vie déstabilisants, avec parfois des conséquences sociales, psychologiques, financières, matérielles et physiques (Mortensen, Latimer et Yusuf, 2014; Neely-Barnes et Dia, 2008; Stewart et al., 2008). À ce propos, les écrits scientifiques révèlent une sous-utilisation des services de santé et de soutien chez les personnes immigrantes, malgré leurs besoins d'aide (Baker, Miller, Dang, Yaangh et Hansen, 2010; Khanlou et al., 2014; Stewart et al., 2008). Les raisons en sont principalement des lacunes sur le plan de la maîtrise de la langue du pays d'accueil ainsi que le manque d'informations au sujet du fonctionnement du réseau de la santé, des services sociaux et des structures d'aide (Jennings, Khanlou et Su, 2014; King, Esses et Solomon, 2013; Mortensen et al., 2014). De surcroît, la distance socioculturelle entre les parents et les professionnels (santé, social, éducation) peut entraîner des 
incompréhensions quant aux rôles de chacun auprès de l'enfant (Daudji et al., 2011; Harry, 2008).

En rapport avec les attentes et les besoins de soutien des parents immigrants, des auteurs soulignent que les difficultés rencontrées se modifient au fil du temps (Harry, 2008; Lin, Macmillan et Brown, 2010). Ce constat confirme la nécessité d'un accompagnement sur mesure lors des différentes étapes de la vie familiale. Ce thème reste toutefois peu abordé dans les écrits scientifiques (King et al., 2013). La présente étude vise à explorer la mobilisation parentale des ressources de soutien formel jusqu'à l'âge adulte de leur enfant en situation de handicap. Afin de mieux comprendre les particularités de leur contexte de vie, le modèle de stress et de coping du double ABCX de McCubbin et Patterson (1983) est utilisé pour appréhender les multiples sources de tension et les ressources utilisées.

\section{Modèle théorique : stress et coping}

Compte tenu de la diversité et de la complexité des parcours de ces parents immigrants, l'utilisation d'un modèle théorique intégrant l'ensemble de leurs expériences dans une perspective dynamique s'avère appropriée. Selon le modèle du double $\mathrm{ABCX}$ de McCubbin et Patterson (1983), (a) une situation de stress (annonce du diagnostic de l'enfant, par exemple) peut déstabiliser les parents. Or, (b) leurs ressources, autant personnelles (maîtrise de la langue du pays d'accueil, par exemple) qu'extérieures (soutien financier pour les besoins particuliers de l'enfant, par exemple), ne permettent pas toujours d'y faire face. Si (c) les circonstances sont considérées comme menaçantes (stigmatisation par la communauté d'origine, par exemple), (x) une crise (dépression, séparation du couple, par exemple) peut éventuellement survenir. 
Toujours en se basant sur le modèle de McCubbin et Patterson (1983), il ressort qu'avec le temps, (aA) l'accumulation des défis (trajectoire migratoire, ajustement au diagnostic, par exemple) requiert la mobilisation (bB) des ressources acquises lors de crises antérieures, additionnées aux moyens personnels existants (capacités d'adaptation, par exemple). Selon (cC) la perception de la situation (interprétation du diagnostic de l'enfant, par exemple), les parents atteignent un nouvel état d'équilibre, soit satisfaisant (adaptation réussie), soit dysfonctionnel (épuisement du parent, par exemple), les menant alors à (xX) une crise supplémentaire. À ce sujet, des auteurs estiment que le soutien social peut favoriser la résolution de ces bouleversements (Gouin, da Estrela, Desmarais et Barker, 2016; Guralnick, Hammond, Neville et Connor, 2008).

Le modèle du double ABCX (McCubbin et Patterson, 1983) a été intégré dans plusieurs études sur les parents d'un enfant en situation de handicap (Hall et Graff, 2011; Pozo, Sarriá et Brioso, 2014; Xue, Ooh et Magiati, 2014). Il tient compte des changements qui s'opèrent avec le temps et il permet d'explorer comment les ressources parentales peuvent être consolidées grâce au soutien formel.

\section{Sources de stress des parents immigrants}

Pour les parents immigrants d'un enfant avec un retard de développement, les sources potentielles de stress s'avèrent multiples. Trois causes principales ressortent de la majorité des écrits scientifiques consultés, à savoir: (1) la langue et la culture, (2) les aspects financiers et matériels, (3) le réseau social.

Suite à l'arrivée dans un nouveau pays, le manque de maîtrise de la langue du pays d'accueil (le cas échéant) et les divergences culturelles constituent une première source de 
stress pour les personnes immigrantes (Baker et al., 2010; Jegatheesan, Miller et Fowler, 2010; Stewart et al., 2008). Ne pas comprendre la langue complexifie la communication avec les professionnels de la santé, du social et de l'éducation (Khanlou et al., 2014). Ceci s'illustre également lors de l'installation familiale, comme dans la recherche d'un logement ou d'un emploi (Stewart et al., 2008). De surcroît, à cause de la situation particulière de l'enfant, les parents immigrants se trouvent souvent confrontés à des démarches administratives complexes et un système de santé inconnu (Khanlou et al., 2014; Mortensen et al., 2014). Des événements stressants s'observent également lors de l'annonce du diagnostic, avec l'utilisation d'un langage spécialisé par les professionnels qui rend la compréhension de la condition de l'enfant encore plus compliquée (Khanlou, Mustafa, Vazquez, Haque et Yoshida, 2015; Lawton, Gerdes, Haack et Schneider, 2014). Il arrive que l'interprétation parentale du diagnostic et la perception des conséquences des besoins particuliers de l'enfant divergent de celles des professionnels (Baker et al., 2010; Jegatheesan et al., 2010). Par conséquent, lorsque les valeurs, les croyances et les attitudes des parents immigrants croisent celles des intervenants (santé, social, éducation), des désaccords surgissent parfois et peuvent provoquer des situations stressantes (Daudji et al., 2011; Mortensen et al., 2014).

Une deuxième source majeure de stress concerne la précarité financière et matérielle de la famille (Khanlou et al., 2014; Khanlou et al., 2015). D’une part, les personnes immigrantes rencontrent habituellement plus de difficultés à intégrer le marché de l'emploi, malgré leur niveau de formation souvent élevé (Côté, 2013; Stewart et al., 2008). D’autre part, prendre soin d'un enfant avec des besoins spéciaux peut entrainer une diminution du temps de travail, voire la décision de quitter son emploi pour l'un des parents afin de se consacrer 
davantage à l'enfant (Khanlou et al., 2015). Cette vulnérabilité financière s'amplifie avec les coûts liés aux soins spécialisés (Bourke-Taylor, Cotter et Stephan, 2014). En outre, le statut légal des parents dans le pays d'accueil représente un facteur de stress, surtout pour les personnes réfugiées qui font face à divers obstacles pour accéder à des programmes de soins, de soutien ou d'éducation (King et al., 2013; Riggs et al., 2012; Stewart et al., 2008).

L'isolement social constitue la troisième source majeure de stress. Considérant les différentes trajectoires migratoires, il arrive que les parents peinent à mobiliser leur réseau social, la famille élargie restant habituellement dans le pays d'origine (Ben-Cheikh et Rousseau, 2013; Stewart et al., 2008). De surcroît, les parents peuvent manquer d'opportunités de socialisation, d'autant plus lorsqu'ils s'occupent à temps plein de leur enfant (Khanlou et al., 2015). Par ailleurs, la stigmatisation du parent et de l'enfant par la communauté devient quelques fois une source de stress, qui contribue à restreindre le réseau social (Ben-Cheikh et Rousseau, 2013; Khanlou et al., 2015; Zuckerman et al., 2014). Quant au couple, il traverse souvent une situation de crise suite à l'annonce du diagnostic de l'enfant, limitant parfois l'intimité et le temps partagé ensemble (Khanlou et al., 2015). Dans ce cas, l'autre conjoint, qui représente quelquefois la seule personne avec qui partager ses difficultés quotidiennes, s'éloigne, ce qui accentue l'isolement social. En résumé, il ressort des écrits consultés que les parents immigrants d'un enfant en situation de handicap sont soumis à davantage de situations de stress $(\mathrm{a}, \mathrm{aA})$ et présentent des ressources $(\mathrm{b}, \mathrm{bB})$ plus restreintes pour y faire face, en comparaison aux autres parents. 


\section{Ressources formelles des parents immigrants}

Selon le modèle du double ABCX de McCubbin et Patterson (1983), les ressources personnelles et extérieures $(b, b B)$ permettent généralement de faire face à des situations de crise et de s'y adapter de manière fonctionnelle. Provenant d'un réseau informel (famille élargie, amis, voisins, par exemple) ou formel (intervenants d'institutions ou d'associations, regroupements de parents, par exemple), le soutien social constitue une ressource externe qui atténue les répercussions d'événements stressants (Gouin et al., 2016; Guralnick et al., 2008). Dans le cas des parents immigrants, le réseau social assurant le soutien informel s'amenuise avec la trajectoire migratoire. De surcroît, le soutien formel semble moins accessible (méconnaissances de la langue et des services, par exemple) (Khanlou et al., 2014; Khanlou et al., 2015; Stewart et al., 2008).

De nombreux auteurs abordent les besoins des parents d'un enfant en situation de handicap (Abbott et Jessiman, 2015; Almarsi, O’Neil et Palisano, 2014; Alsem et al., 2014; Gouin et al., 2016; Hodgetts et al., 2015). Par contre, peu de recherches recensées explorent le contexte des parents immigrants. Au Canada, l'étude de Stewart et ses collaborateurs (2008) illustre les difficultés des personnes immigrantes et réfugiées nouvellement arrivées (sans enfant en situation de handicap), notamment en matière d'accès aux services formels. Quant aux travaux canadiens de Khanlou et ses collaborateurs $(2014,2015)$ ainsi que ceux de Jennings et ses collaboratrices (2014), ils documentent les défis rencontrés respectivement par les pères et les mères immigrants d'un enfant en situation de handicap, en termes de soutien et de politiques sociales. Le manque de ressources d'aide informelle et les barrières d'accès aux services formels ressortent de ces trois recherches, sans toutefois que les parents soient consultés. 
Pour la présente étude, la parole est donnée aux parents immigrants sur leurs attentes et leurs besoins en ressources de soutien formel. Une typologie de ces stratégies de soutien, adaptée des travaux de Tétreault et de ses collaborateurs (2014), est utilisée pour établir une compréhension commune avec les parents contactés. Dix catégories sont distinguées: soutien en information (condition de l'enfant, services existants); accompagnement à la décision (soutien administratif); soutien juridique; soutien financier (compensations, aide aux projets); soutien en éducation (interactions avec l'enfant); écoute active; assistance au quotidien (aide physique); soutien pour les loisirs (accompagnement pour le sport et les activités sociales); soutien au transport (utilisation, adaptation); répit, garde de l'enfant (activités habituelles) ou dépannage (situations inhabituelles).

Dans cette perspective, les situations de stress vécues par les parents immigrants, ainsi que leurs attentes et leurs besoins en termes de ressources de soutien formel seront analysés. Par ailleurs, les catégories d'aide formelle utilisées durant l'année antérieure et l'appréciation parentale de ce recours seront examinées. Enfin, une discussion des résultats permettra d'explorer de manière systémique et dynamique le contexte des parents immigrants avec l'enfant qui vieillit.

\section{Méthode}

Afin d'explorer leurs attentes et leurs besoins en soutien formel, des entrevues semidirigées ont été menées auprès de parents immigrants d'un enfant en situation de handicap. Considérant les enjeux éthiques et la sensibilité du sujet abordé, l'utilisation d'une approche qualitative exploratoire auprès d'un petit échantillon est justifiée. De plus, ce choix méthodologique correspond à une thématique peu étudiée et constitue une option 
privilégiée afin de documenter la perception des parents (Imbert, 2010). En outre, la possibilité qu'offrent les entrevues de clarifier les questions et les réponses, le cas échéant, convient spécifiquement à des rencontres interculturelles. Le projet a reçu l'approbation du Comité d'éthique de la recherche de l'Institut de réadaptation en déficience physique de Québec (IRDPQ), Québec, Canada.

Les parents ont été sollicités par l'intermédiaire d'organismes communautaires et de centres de santé et de services sociaux offrant des services à ces personnes. Les critères d'inclusion étaient (1) d'avoir un enfant en situation de handicap, âgé entre deux et dix-sept ans, (2) d'être une personne immigrante de première génération installée au Québec depuis au moins deux ans (ville de Québec ou de Montréal), (3) d'avoir reçu des services en lien avec les difficultés de l'enfant, et (4) de pouvoir s'exprimer en français. Afin de documenter l'évolution des attentes et des besoins en soutien formel, les participants ont été recrutés de manière à obtenir une diversité des âges des enfants. Les parents intéressés recevaient un feuillet d'information sur le projet ainsi qu'un formulaire de consentement avant la rencontre. Les entrevues étaient organisées soit au domicile du parent, soit dans un lieu de leur choix, selon leurs disponibilités. Les échanges, d'une heure environ, étaient enregistrés, puis retranscrits.

Un guide d'entretien permettait le maintien d'une structure similaire entre les différentes rencontres (Miles et Huberman, 2003). Une première section avec des questions ouvertes portait sur les attentes et les besoins des participants en matière de services de soutien formel. Ceux-ci devaient être destinés aux parents pour leur permettre d'atteindre un équilibre dans toutes les sphères de leur vie. La deuxième section du guide servait à documenter les dix types de stratégies de soutien formel mobilisés durant l'année 
antérieure. Pour chacun d'entre eux (adaptées de Tétreault et al., 2014), une définition était proposée aux participants. Les parents indiquaient (1) s'ils avaient reçu ce soutien durant l'année écoulée, de la part de qui et sous quelle forme, puis (2) si ce soutien avait répondu à leurs attentes. Ils étaient invités à ajouter les raisons pour lesquelles certains types d'aide n'avaient pas répondu à leurs attentes et à proposer des améliorations. En fin d'entrevue, un questionnaire sur les données sociodémographiques du parent et de l'enfant avec des besoins particuliers était rempli.

Concernant les propos des parents, deux types d'analyses qualitatives ont été appliqués. Premièrement, une analyse thématique inductive du corpus de données a permis d'en extraire les situations évoquées par les participants comme constituant des sources de stress qui pourraient nécessiter le recours au soutien formel (Miles et Huberman, 2003; Paillé et Mucchielli, 2012). Ces situations, perçues comme menaçantes par les parents, sont explorées à travers le modèle du double ABCX (McCubbin et Patterson, 1983). Le regroupement et la hiérarchisation de ces sources de tension se sont déroulés de manière itérative, en consultant les coauteures. Deuxièmement, une analyse par questionnement analytique a guidé le traitement du discours lié au soutien formel utilisé et à son appréciation par les parents (Paillé et Mucchielli, 2012). Les ressources parentales (b, bB) permettant de faire face aux situations de stress (a, aA) ont été analysées. Lors de la discussion des résultats, une représentation théorique du contexte particulier des parents immigrants d'un enfant en situation de handicap est formulée. Pour les données sociodémographiques, une analyse descriptive est présentée. 


\section{Résultats}

Suite à la description des huit participants à cette étude, une présentation des attentes et des besoins des parents est faite. Elle met en évidence les sources de tensions (a, aA) nommées par les participants et la manière dont ils les perçoivent (c, cC). Puis, les services de soutien formel (b, bB) utilisés durant l'année antérieure sont identifiés, en y associant l'appréciation que les parents en ont.

\section{Caractéristiques des participants}

La description des caractéristiques sociodémographiques des deux pères et des six mères, représentant huit familles différentes, se trouve dans le tableau 1. Il s'avère qu'aucun parent n’occupe d'activité professionnelle rémunérée au moment de l'entrevue.

Tableau 1. Description des huit parents rencontrés.

\begin{tabular}{|c|c|c|c|c|c|c|}
\hline Code & $\begin{array}{c}\text { Sexe } \\
\text { Tranche } \\
\text { d'âges }\end{array}$ & $\begin{array}{l}\text { Situation } \\
\text { familiale }\end{array}$ & $\begin{array}{l}\text { Niveau de } \\
\text { formation }\end{array}$ & $\begin{array}{l}\text { Région } \\
\text { d'origine }\end{array}$ & $\begin{array}{c}\text { Arrivée au } \\
\text { Canada } \\
\text { Type } \\
\text { d'immigratio } \\
n \\
\end{array}$ & $\begin{array}{l}\text { Âge enfant } \\
\text { en } \\
\text { situation } \\
\text { de } \\
\text { handicap }\end{array}$ \\
\hline $\mathrm{P} 1$ & $\begin{array}{c}\text { Femme } \\
21-40\end{array}$ & En couple & $\begin{array}{c}\text { Universitair } \\
\mathrm{e}\end{array}$ & $\begin{array}{l}\text { Afrique du } \\
\text { Nord }\end{array}$ & $\begin{array}{c}<5 \text { ans } \\
\text { Économique }\end{array}$ & 2 ans \\
\hline $\mathrm{P} 2$ & $\begin{array}{c}\text { Femme } \\
21-40\end{array}$ & En couple & $\begin{array}{c}\text { Universitair } \\
\mathrm{e}\end{array}$ & $\begin{array}{l}\text { Afrique du } \\
\text { Nord }\end{array}$ & $\begin{array}{c}<5 \text { ans } \\
\text { Économique }\end{array}$ & 3 ans \\
\hline P3 & $\begin{array}{c}\text { Femme } \\
21-40\end{array}$ & En couple & $\begin{array}{c}\text { Universitair } \\
\mathrm{e}\end{array}$ & $\begin{array}{l}\text { Afrique du } \\
\text { Nord }\end{array}$ & $\begin{array}{c}\text { 5-10 ans } \\
\text { Economique }\end{array}$ & 3 ans \\
\hline P4 & $\begin{array}{c}\text { Homme } \\
61+\end{array}$ & En couple & $\begin{array}{l}\text { Secondaire } \\
\text { supérieur }\end{array}$ & Europe & $\begin{array}{c}>10 \text { ans } \\
\text { Économique }\end{array}$ & 7 ans \\
\hline P5 & $\begin{array}{c}\text { Femme } \\
41-60\end{array}$ & Seule & Secondaire & Caraïbes & $\begin{array}{c}>10 \text { ans } \\
\text { Économique }\end{array}$ & 10 ans \\
\hline P6 & $\begin{array}{c}\text { Femme } \\
21-40\end{array}$ & En couple & Secondaire & $\begin{array}{l}\text { Amérique } \\
\text { du Sud }\end{array}$ & $\begin{array}{l}<5 \text { ans } \\
\text { Refuge }\end{array}$ & 11 ans $^{*}$ \\
\hline P7 & $\begin{array}{c}\text { Homme } \\
41-60\end{array}$ & En couple & $\begin{array}{c}\text { Universitair } \\
\mathrm{e}\end{array}$ & $\begin{array}{l}\text { Amérique } \\
\text { du Sud }\end{array}$ & $\begin{array}{l}>10 \text { ans } \\
\text { Refuge }\end{array}$ & 12 ans \\
\hline
\end{tabular}




$\begin{array}{cccccc}\text { P8 } & \begin{array}{c}\text { Femme } \\ 21-40\end{array} & \text { Seule } & \begin{array}{c}\text { Secondaire } \\ \text { supérieur }\end{array} & \begin{array}{c}\text { Afrique du } \\ \text { Nord }\end{array} & \begin{array}{c}<5 \text { ans } \\ \text { Refuge }\end{array}\end{array}$

\section{*Enfant né avant l'immigration.}

À la lecture du tableau 1, il ressort que quatre mères vivent en couple et deux autres demeurent seules avec leur enfant. Cinq participantes sont âgées de 21 à 40 ans et une de 41 à 60 ans. Trois mères possèdent une formation de niveau universitaire, alors que deux femmes ont un diplôme de niveau secondaire et une autre de secondaire supérieur. Quatre participantes sont originaires de différents pays d'Afrique du Nord, une mère des Caraïbes et une autre d'Amérique du Sud. Seulement deux femmes ont un statut de réfugié à l'arrivée au Canada, tandis que les quatre autres sont entrées dans le pays d'accueil comme immigrantes économiques. Quatre mères demeurent depuis moins de cinq ans au Canada, une depuis cinq à dix ans, et une autre a quitté son pays d'origine il y a plus de dix ans.

Les deux pères rencontrés vivent chacun en couple. Un participant a entre 41 et 60 ans et le deuxième plus de 60 ans. Un des pères vient d'Europe et possède un diplôme de niveau secondaire supérieur. Le second, de formation universitaire, a quitté l'Amérique du Sud pour rejoindre le Canada en tant que réfugié. Ils demeurent tous deux au Canada depuis plus de dix ans.

L'âge des enfants en situation de handicap se situe entre 2 et 16 ans (moyenne de 8 ans). Ils présentent des atteintes développementales variables. Il faut noter que les parents ont parfois de la difficulté à préciser le diagnostic et ne l'évoquent pas toujours clairement lors de l'entrevue. Néanmoins, tous les enfants ont reçu des services de santé en lien avec leurs difficultés spécifiques. 


\section{Sources de stress}

Dans un premier temps, les parents rencontrés se sont exprimés sur leurs attentes et leurs besoins en ce qui concerne le soutien formel pour eux-mêmes. Sept sources de stress (a, aA) ressortent de l'analyse thématique de leur discours comme étant un problème à leurs yeux (c, cC). Ces tensions sont en lien avec (1) le parcours migratoire, (2) la situation de handicap de l'enfant, (3) l'organisation sociosanitaire du pays d'accueil, (4) l'insertion professionnelle, (5) la relation de couple, (6) le réseau social et (7) l'état de santé du parent. Ces différentes sources de stress sont abordées séparément, bien que la plupart des parents en rencontrent plus d'une simultanément.

\section{Parcours migratoire}

Suivant le parcours migratoire vécu par les parents (événements traumatisants, conditions d'entrée au Canada, par exemple), les attentes et les besoins exprimés varient. Pour tous, l'intérêt de l'enfant est prioritaire. Néanmoins, alors que la plupart des répondants ont aussi des attentes à l'égard du soutien qui leur est adressé, le discours de quelques parents, généralement arrivés comme réfugiés, se concentre sur l'aide à l'enfant, en particulier lorsqu'il est né avant l'immigration. Dans ce sens, une mère explique qu'elle ne manque de rien et que tous ses espoirs d'un avenir meilleur reposent sur le soutien destiné à sa fille adolescente. De même, une autre participante déclare : "J'ai besoin, c'est vrai, que pour [mon enfant], pas pour moi, beaucoup plus. Moi, j'ai vécu ma vie. J'ai terminé. » (P8, mère d'une adolescente). 


\section{Situation de handicap}

Que le diagnostic ait déjà été annoncé ou que l'évaluation soit en cours, les parents rencontrés observent et reconnaissent les difficultés de leur enfant. Lorsque ce dernier est encore très jeune, le choc de cette annonce reste parfois manifeste et des incertitudes demeurent quant à sa condition de santé. Des besoins en matière d'information s'avèrent souvent formulés, comme pour cette mère dont l'adolescente est née dans le pays d'origine : «Moi, je suis toujours en attente. Ma fille, elle est toujours en évaluation. » (P8, mère d'une adolescente). La répondante explique qu'elle ne connaissait pas toutes les difficultés de sa fille avant d'arriver au Canada. Pour d'autres, l'évolution de la situation de l'enfant et sa réévaluation constituent une source actuelle de stress, comme l'évoque ce père : «Mais c'est important parce que si on ne connaît pas qu'est-ce qui arrive à l'enfant, on ne sait pas comment on peut réagir. »( $\mathrm{P} 7$, père d'un adolescent).

\section{Organisation sociosanitaire du pays d'accueil}

Lorsque les parents immigrants vivent dans un nouvel environnement tout en prenant soin de leur enfant, il arrive que l'organisation du pays d'accueil (santé, services sociaux, éducation, cadre juridique) constitue une source de stress. À ce propos, un participant souligne son incompréhension des interventions de réadaptation disponibles à l'école sur la base de subventions étatiques: «Le gouvernement du Québec, par exemple, il donne des montants dédiés pour l'enfant. Mais l'école ne les utilise pas pleinement. ... Mais là, on se sent un petit peu trahi... » ( $\mathrm{P} 4$, père d'un garçon). Ce sentiment de trahison entraîne des tensions chez ce père, qui désire davantage de services d'orthophonie pour son garçon. Pour une autre participante, la recherche d'un logement convenable constitue un problème majeur. Ses attentes reposent sur la reconnaissance de l'aspect prioritaire de sa situation : 
«Dans l'administration, les gens sont là pour s'occuper des gens qui viennent immigrer ici, au Québec. Ils donnent des services, c'est vrai. Mais il y en a qui ne savent pas qu'estce qui se passe à l'intérieur... » ( $\mathrm{P} 5$, mère d'une adolescente).

\section{Insertion professionnelle}

Lors de l'arrivée dans le pays d'accueil ou suite à un congé parental, des répondants envisagent d'intégrer (ou de réintégrer) le marché de l'emploi au Québec ou de commencer une formation. Ces projets ne voient pas forcément le jour, en particulier lorsqu'ils se trouvent très impliqués auprès de leur enfant et de son programme de réadaptation. La mère d'un très jeune enfant explique : "Tout s'est arrêté d'un seul coup. ... Je ne travaille plus alors que j'étais une femme très active. ... Puis, tout à coup, je suis femme au foyer avec un enfant très malade... » (P1, mère d'un très jeune garçon). Les parents décident souvent de différer leur retour en emploi ou aux études. Reprendre une activité professionnelle apparaît pour eux comme une source de stress, car l'emploi représente une ressource financière, un bris de l'isolement et une valeur sociale. Une participante s'exprime à ce propos : «Parfois, ça me tente de retourner chez moi. C'est comme si ça me manque d'être une femme active. J'ai un rôle social. » (P1, mère d'un très jeune garçon).

\section{Relation de couple}

Du point de vue de quelques participants, l'annonce du diagnostic ou le constat des difficultés de l'enfant a déstabilisé la relation avec leur partenaire. En effet, le processus d'acceptation des particularités de l'enfant se déroule parfois à des rythmes différents chez les deux parents, comme l'illustre cette répondante: "Au début, mon mari, il n'a pas accepté le diagnostic. ... Donc, ça a causé beaucoup de tensions à la maison. » (P2, mère 
d'un très jeune garçon). Il arrive que les changements de rôles, à la fois professionnels et familiaux, demandent une adaptation pour les parents. Lorsqu'un seul conjoint subvient aux besoins de la famille, des tensions peuvent apparaître au sein du couple en raison du partage des tâches à accomplir à domicile (prendre soin de l'enfant, s'occuper des tâches ménagères, par exemple) ou à cause de problèmes financiers. Une femme note à ce propos : «C'est juste mon mari qui travaille, et un travail autonome, presque saisonnier. ... Ce qui fait qu'à un certain moment, le manque d'argent, c'est horrible. » ( $\mathrm{P} 3$, mère d'un très jeune garçon). De surcroît, les décisions concernant l'éducation peuvent mener à des désaccords dans le couple. Face à ces difficultés conjugales, le besoin d'un soutien psychologique est parfois évoqué.

\section{Réseau social}

L'isolement social est mis en évidence par plusieurs parents. Il peut être causé par les difficultés spécifiques de l'enfant, comme l'illustre cette participante : "Les personnes que je connais, à cause de la situation, ne sont pas compréhensives... . Je les ai perdues une à une. » ( $\mathrm{P} 3$, mère d'un très jeune garçon). Il découle aussi de la trajectoire migratoire, considérant l'éloignement de la famille élargie. Avec un tissu social restreint, les parents mettent en évidence des besoins en soutien qui ne peuvent être comblés par le réseau informel (famille, amis, voisins, collègues de travail). Selon la culture d'origine, la famille joue un rôle particulièrement important, ce qui renforce le poids de l'absence, comme l'affirme cette femme: «Parce que pour nous, c'est une habitude qu'on reste ensemble tout le temps. Quand, dans notre pays, notre culture, c'est que la famille reste tout le temps avec les enfants. » ( $\mathrm{P} 6$, mère d'une adolescente). 
État de santé du parent

Des parents décrivent comment des problèmes de santé peuvent diminuer l'énergie disponible pour assurer leurs responsabilités auprès de l'enfant, ainsi que le mentionne ce père : «Là, moi-même, j'ai une liste de problèmes de santé, ok? Donc j'essaie de ne pas alourdir.» (P4, père d'un garçon). Plusieurs participants évoquent des épisodes de dépression. Néanmoins, ils restent centrés sur les services qu'ils pourraient recevoir pour leur enfant plutôt que sur le soutien qui leur est destiné, tel que le note ce répondant: «Mais je pense, ce qui me donnerait l'énergie, c'est vraiment de voir que l'enfant est pris en charge de la bonne façon dans son domaine... » (P4, père d'un jeune garçon). Leur état de santé ne semble pas constituer leur plus grande source d'inquiétudes. Face à l'ensemble des situations de stress présentées, les parents peuvent recourir à différentes ressources de soutien formel.

\section{Ressources de soutien formel}

La plupart des participants disent recevoir du soutien formel dans différents domaines (accompagnement dans les démarches administratives, écoute active, service de garde, par exemple). Ces ressources externes $(b, b B)$ leur permettent de combler une partie de leurs attentes et leurs besoins d'aide afin de faire face au cumul de situations stressantes (a, aA). Toutefois, des parents identifient des difficultés d'accès à divers services (répit, écoute active, par exemple) ou de l'insatisfaction vis-à-vis de l'aide reçue (soutien en éducation, accompagnement à la décision, par exemple).

Tous les parents rencontrés se sont exprimés sur leur recours aux dix stratégies de soutien formel durant l'année écoulée. Selon les résultats présentés dans le tableau 2, le soutien 
financier constitue la seule stratégie mentionnée par les huit parents interrogés. De plus, le soutien en information, l'accompagnement à la décision et le soutien en éducation ont servi à sept des huit participants durant la même période. Quant aux ressources pour le transport, six parents en ont bénéficié. L'écoute active et le soutien aux loisirs, tout comme le répit, la garde de l'enfant et le dépannage, ont chacun été utilisés par cinq participants lors des 12 derniers mois. Par contre, les parents contactés ont moins eu recours au soutien juridique $(n=2)$ et à l'aide au quotidien $(n=1)$. Les raisons du recours (ou non) à ces différentes stratégies ainsi que l'appréciation des parents concernant l'aide reçue sont décrites par catégorie sur la base des propos recueillis.

Tableau 2. Utilisation par les parents des stratégies de soutien formel durant l'année écoulée ( $\mathrm{X}=$ utilisé $)(\mathrm{N}=8)$.

\begin{tabular}{|c|c|c|c|c|c|c|c|c|c|}
\hline \multirow{2}{*}{$\begin{array}{l}\text { Dix stratégies } \\
\text { de soutien } \\
\text { formel }^{*}\end{array}$} & \multirow{2}{*}{$\begin{array}{c}\text { Nombre } \\
\text { d'utilisateur } \\
\text { S } \\
\text { N }\end{array}$} & \multicolumn{8}{|c|}{ Code du parent } \\
\hline & & P4 & P1 & P2 & P3 & P6 & P7 & P8 & P5 \\
\hline Soutien financier & 8 & $X$ & $\mathrm{X}$ & $\mathrm{X}$ & $\mathrm{X}$ & $\mathrm{X}$ & $\mathrm{X}$ & $\mathrm{X}$ & $X$ \\
\hline $\begin{array}{l}\text { Soutien en } \\
\text { information }\end{array}$ & 7 & & $\mathrm{X}$ & $\mathrm{X}$ & $\mathrm{X}$ & $\mathrm{X}$ & $\mathrm{X}$ & $\mathrm{X}$ & $\mathrm{X}$ \\
\hline $\begin{array}{l}\text { Accompagnement } \\
\text { à la décision }\end{array}$ & 7 & $X$ & $\mathrm{X}$ & $X$ & $X$ & $X$ & & $\mathrm{X}$ & $\mathrm{X}$ \\
\hline Soutien en éducation & 7 & $X$ & $\mathrm{X}$ & $\mathrm{X}$ & $\mathrm{X}$ & $\mathrm{X}$ & $\mathrm{X}$ & & $\mathrm{X}$ \\
\hline Soutien au transport & 6 & $X$ & $\mathrm{X}$ & & & $\mathrm{X}$ & $X$ & $X$ & $X$ \\
\hline Écoute active & 5 & & $X$ & $\mathrm{X}$ & $\mathrm{X}$ & & & $X$ & $X$ \\
\hline Soutien aux loisirs & 5 & $\mathrm{X}$ & & $\mathrm{X}$ & & & $\mathrm{X}$ & $\mathrm{X}$ & $\mathrm{X}$ \\
\hline $\begin{array}{l}\text { Répit, garde, } \\
\text { dépannage }\end{array}$ & 5 & & & & $X$ & $X$ & $\mathrm{X}$ & $\mathrm{X}$ & $\mathrm{X}$ \\
\hline Soutien juridique & 2 & & & & & & & $\mathrm{X}$ & $\mathrm{X}$ \\
\hline $\begin{array}{l}\text { Assistance au } \\
\text { quotidien }\end{array}$ & 1 & & & & & & & & $\mathrm{X}$ \\
\hline \multicolumn{2}{|c|}{$\begin{array}{l}\text { Nombre de stratégies } \\
\text { de soutien mobilisées }\end{array}$} & 5 & 6 & 6 & 6 & 6 & 6 & 8 & 10 \\
\hline
\end{tabular}

*Adapté de Tétreault et al. (2014). 


\section{Soutien financier}

Tous les participants reçoivent un soutien financier et la plupart s'avèrent satisfaits de l'aide obtenue. Néanmoins, certains considèrent que les sommes versées ne suffisent pas à couvrir les coûts liés aux besoins spécifiques de l'enfant (thérapies supplémentaires ou privées, gardiennage, par exemple). Des parents sollicitent parfois le soutien en argent auprès de fondations ou d'autres institutions caritatives, bien que les listes d'attente soient longues : "On a eu une aide, par exemple, de cet organisme-là, une fois. Ils nous ont payé carrément un mois de loyer. " (P1, mère d'un petit garçon de 2 ans). Néanmoins, des participants expriment une gêne à demander du soutien financier. Les préoccupations dans ce domaine constituent parfois un motif pour reprendre une activité professionnelle, comme l'illustre cette mère: "Vu qu'elle grandit, c'est vraiment. C'est pour ça que je veux travailler, moi. C'est pour ça que je veux avoir un travail fixe et puis stable, à temps plein... . » (P8, mère d'une adolescente).

\section{Soutien en information}

La plupart des parents se disent satisfaits des informations reçues de la part des professionnels de la santé en ce qui concerne la condition de leur enfant. Certains restent dans l'attente d'un diagnostic ou d'une réévaluation de l'enfant qui vieillit. Des renseignements complémentaires prennent quelquefois une importance particulière, notamment lorsqu'une nouvelle grossesse est souhaitée, car le risque d'un problème lié à l'hérédité peut exister : «Puis j'aspire le plus tôt possible... [à en] avoir un deuxième... . Puis là, on attend. ... C'est vraiment une note d'espoir, comme ça, pour nous... . » (P1, mère d'un très jeune garçon). Concernant les services disponibles, l'information semble généralement bien relayée, sauf dans certains cas où le transfert de connaissances se fait 
difficilement et n'arrive pas au moment désiré par le parent. Selon des participants, les changements fréquents d'intervenants perturbent la transmission des informations.

Accompagnement à la décision

Les parents évoquent souvent les travailleurs sociaux comme ressource principale d'accompagnement à la décision. Obtenir des références pour des services, de l'aide dans les démarches administratives et des conseils quant à la scolarisation de l'enfant constitue le type de soutien dont ils se disent satisfaits. Malgré ce constat, il semble que des besoins persistent, notamment lorsque la capacité d'agir des parents se trouve diminuée par la fatigue et les tâches quotidiennes, comme le rapporte cette mère : "C'est plus la motivation et la persévérance, vraiment. J'ai besoin d'un petit coup de pouce, là, ou de l'aide, là, pour aller vers l'avant. " (P3, mère d'un très jeune garçon). L'insatisfaction parentale peut aussi provenir d'incompréhensions avec les professionnels (distance ressentie avec l'intervenant, manque de suivi, par exemple). Pour d'autres participants, solliciter de l'accompagnement dans les démarches entraîne une gêne, comme l'indique cette mère : "Parce que ça fait longtemps qu'on fait les choses nous, seuls. ... Parce qu'on n'aime pas déranger les autres personnes. » (P6, mère d'une adolescente).

\section{Soutien en éducation}

La plupart des personnes rencontrées sont satisfaites du soutien reçu en éducation. «Ça, c'est un service, vraiment, qui m'a aidée beaucoup. ... [L'éducatrice] m'outillait par les trucs, comment je me comporte avec mon fils. » (P3, mère d'un très jeune garçon). Parfois, les parents considèrent que l'aide proposée ne leur apporte pas de nouvelles connaissances ou ne correspond pas à leurs attentes. L'entrée dans l'adolescence peut aussi perturber la 
relation entre le parent et l'enfant, demandant davantage de soutien lorsque la communication est difficile, comme pour cette mère et sa fille : "Elle se renferme. Elle rentre dans sa bulle. ... Elle ne communique pas. » (P8, mère d'une adolescente).

\section{Soutien au transport}

Souvent proposé par le travailleur social, le soutien aux déplacements se concrétise notamment par le transport adapté. Lorsque l'enfant est encore jeune ou selon sa situation de handicap, les parents ne requièrent pas ce type d'aide. En outre, quelques participants déclarent obtenir un dédommagement financier pour le coût des déplacements et du stationnement lors des multiples consultations de leur enfant. Néanmoins, ce soutien ne s'avère pas connu de tous les parents.

\section{Écoute active}

Lorsque les liens avec la famille élargie restée au pays d'origine demeurent solides, les parents bénéficient habituellement d'une écoute informelle grâce aux technologies de communication. Cependant, certains estiment devoir protéger leur entourage des tracas liés à leur quotidien. D’autres parents recourent à de l'écoute formelle, notamment de la part des travailleurs sociaux ou des intervenants d'associations, et s'en disent satisfaits. Toutefois, pour quelques participants, l'aide provenant du travailleur social ne correspond pas à leurs attentes, car ils recherchent davantage les services d'un psychologue, comme l'explique cette mère : "Parce que la travailleuse sociale, elle est là pour vous aider et tout, mais elle ne peut pas vous écouter, là, sur le plan psychologique, que ça. » (P1, mère d'un très jeune garçon). Selon cette participante, les services d'un psychologue devraient être proposés à tous les parents immigrants d'un enfant en situation de handicap sans avoir 
à les solliciter. Il est à noter que les rencontres avec d'autres parents d'un enfant en situation de handicap constituent également une forme de soutien jugée utile.

\section{Soutien aux loisirs}

En général, le soutien aux loisirs est utilisé par les parents pour l'accompagnement de l'enfant dans ses activités hors du domicile. Bien que parfois méconnue, cette aide semble être appréciée. Encore dans un processus d'évaluation, cette mère s'exprime dans ce sens : «Comme les loisirs. J'ai hâte. Elle a pris beaucoup de poids. Oui, trop. Je me fais du souci pour elle. Parce que là, je ne peux pas lui faire sa toilette, sa douche. " (P8, mère d'une adolescente). Il arrive néanmoins que l'accompagnement devienne embarrassant pour l'enfant, surtout s'il désire davantage d'indépendance à l'adolescence, incitant les parents à envisager de l'arrêter. Des réticences liées à cet accompagnement et à l'accès à de nouvelles activités pour l'enfant (dormir à l'extérieur du domicile, par exemple) sont aussi soulignées par quelques participants.

\section{Répit, garde et dépannage}

Si aucun participant n'évoque l'utilisation de services de dépannage (soutien en cas d'urgence), le recours aux services de répit et de garde est plus fréquent. En fonction de leurs besoins, les parents reçoivent une subvention de l'État afin d'engager quelqu'un pour s'occuper de l'enfant en situation de handicap lorsqu'ils s'absentent. Parfois, l'accès à ce service arrive de manière décalée par rapport aux besoins des participants. De plus, leurs attentes envers la personne responsable de l'enfant pendant leur absence peuvent ne pas être satisfaites (type de formation, niveau d'expérience, par exemple). De même, l'incompréhension entre les communautés culturelles peut intervenir dans le choix d'une 
gardienne, comme le raconte cette participante : «Il y avait une Québécoise qui s'entendait avec [ma fille] très bien; mais sa mère, elle était contre. ... Là, j'ai décidé que ma fille, la garde provient toujours de la Communauté. » (P8, mère d'une adolescente). Si la plupart des parents privilégient la garde de l'enfant au domicile, pour son bien-être et le leur, trouver un centre de répit à l'extérieur du logement s'avère difficile selon l'âge de l'enfant.

\section{Soutien juridique}

En règle générale, les participants mentionnent ne pas avoir besoin de soutien juridique. Toutefois, peu d'entre eux connaissent l'existence de ce type d'aide, sauf s'ils en ont été informés par un travailleur social, par exemple. Selon les cas, il arrive que du soutien juridique privé soit sollicité.

\section{Assistance au quotidien}

L'assistance au quotidien (tâches ménagères, soins à l'enfant, par exemple) apparaît comme la moins mentionnée. Il ne semble pas s'agir d'un manque de connaissance, mais davantage d'une discordance entre les besoins des parents rencontrés et le type de soutien offert. Dans ce sens, une mère déclare : "Puis, la seule chose qui m’occupait, c'était faire le ménage. Alors ramener quelqu'un pour me le faire, ça serait vraiment déprimant plus qu'aidant. » (P1, mère d'un très jeune garçon). Des participants soulignent leur volonté de réaliser ces tâches eux-mêmes tant qu'ils en sont capables physiquement, notamment par souci que les résultats obtenus correspondent à leurs exigences. De surcroît, l'aide de l'autre parent ou d'amis semble prioritairement sollicitée par rapport à l'aide formelle, comme le précise cette femme : "Mais pour le moment, on n'a pas besoin d'une personne. Parce que [mon 
conjoint] m'aide beaucoup. Chaque fois que je fais une chose, il est avec mes filles. " (P6, mère d'une adolescente).

La qualité de la prise en charge est reconnue, tel que le mentionne cette mère : « ... De toute façon, pour moi, je suis bien prise en charge. ... À chaque fois, j'ai une intervenante vraiment magnifique, à chaque fois. »( $\mathrm{P} 8$, mère d'une adolescente). Les ressources de soutien extérieures $(b, b B)$ constituent un des moyens de faire face au cumul de stress vécu. En résumé, les propos recueillis attestent que malgré l'utilisation de différents types de soutien formel, les attentes et les besoins parentaux ne sont pas toujours entièrement satisfaits, comme le note cette participante : «Là, je suis fatiguée. ... Malgré les aides, malgré que j'ai le répit, malgré ça. Mais il y a beaucoup de choses que je fais moi-même. » ( $\mathrm{P}$ 8, mère d'une adolescente). Les propos de cette mère illustrent la possibilité d'atteindre une situation de crise $(\mathrm{x}, \mathrm{xX})$. Des propositions d'améliorations de l'aide reçue ressortent des entrevues, soit une meilleure collaboration entre les intervenants et la transmission des connaissances acquises par les parents au travers de leurs expériences de vie (parent expert, regroupements de parents, par exemple).

\section{Discussion}

Selon leur parcours, les participants rencontrés avaient vécu des crises dans leur pays d'origine suite à des événements stressants (pays en guerre, violence conjugale, perte d'un enfant, par exemple). Ces situations s'avèrent habituellement plus fréquentes dans le cas de personnes réfugiées. Les écrits scientifiques soulignent l'importance d'en tenir compte lors de l'accompagnement des personnes immigrantes (Lewig, Arney et Salveron, 2010; Mortensen et al., 2014; Stewart et al., 2008). Avec une trajectoire migratoire et des besoins 
particuliers chez l'enfant, de nouvelles sources de tension s'ajoutent aux précédentes. Selon les propos recueillis, les parents dont l'enfant est né dans le pays d'origine et ceux arrivés au Canada en tant que réfugiés semblent mettre plus d'attention sur tout ce qui a trait à l'enfant. Leur propre vie parait avoir peu d'importance, contrairement au discours d'autres parents. Ce constat peut s'expliquer par une réussite du projet migratoire davantage liée à l'avenir de l'enfant, comme l'observent Pondé et Rousseau (2013).

En se basant sur leurs différents rôles (pourvoyeur de soins, conjoint, professionnel, membre de la société), les parents ont évoqué de nombreuses sources de stress. Néanmoins, ils paraissent porter peu d'intérêt à leur état de santé, oubliant leur propre situation pour privilégier les besoins de l'enfant, quel que soit son âge. Ce constat est aussi fait par Lai et Ishiyama (2004) chez des mères immigrantes d'origine chinoise. De surcroît, l'analyse des propos des parents illustre comment la condition de l'enfant entraîne du stress chez la plupart d'entre eux (diagnostic, pronostic, services). Selon les résultats obtenus et en accord avec les écrits scientifiques, ces tensions s'avèrent souvent plus déstabilisantes pour les parents immigrants que pour d'autres, par le cumul des sources de stress, les barrières d'accès aux services (soins, soutien) et par le manque de connaissances du fonctionnement du pays d'accueil (Khanlou et al., 2014; Mortensen et al., 2014; Stewart et al., 2008). Si ces difficultés semblent exister, quel que soit l'âge de l'enfant, les périodes charnières paraissent fragiliser davantage les parents (Lin et al., 2010; Povenmire-Kirk et al., 2010; Weiss, Tint, Paquette-Smith et Lunsky, 2016). Les participants rapportent des sources de tension spécifiquement liées à l'annonce du diagnostic, l'entrée à l'école et la transition à la vie adulte. 
Habituellement déstabilisés par la condition de leur enfant, plusieurs parents rapportent avoir traversé des situations de crise dans leurs relations conjugales à partir de la naissance de l'enfant et plus tard (rôles liés au genre, éducation de l'enfant, par exemple). Des auteurs sont arrivés à ces mêmes constats (Ben-Cheikh et Rousseau, 2013; Khanlou et al., 2015; Stewart et al., 2008). En matière d'insertion professionnelle, les sources de tension varient suivant le but du projet migratoire (emploi, refuge, soins de l'enfant, par exemple), l'âge du parent et son niveau de scolarité. L'ensemble des participants étaient sans emploi lors des entretiens, ce qui illustre les obstacles liés au marché du travail. Des études identifient les mêmes défis, notamment selon le statut d'immigration, le niveau de connaissances de la langue et la reconnaissance des diplômes (Côté, 2013; Stewart et al., 2008).

L'isolement social comme source de stress semble généralisé à l'ensemble des parents rencontrés. Il peut s'agir d'une conséquence de l'immigration, mais aussi de la situation de handicap de l'enfant. Cette observation se retrouve dans d'autres recherches, soulignant le manque d'accès au soutien informel (Ben-Cheikh et Rousseau, 2013; Khanlou et al., 2014; Stewart et al., 2008). Par ailleurs, l'organisation sociosanitaire du pays d'accueil constitue un facteur de stress chez plusieurs participants de la présente étude (recherche d'un logement adapté, attribution de services de réadaptation par l'école, par exemple). D'autres recherches dépeignent ce type de difficultés, en matière de compréhension du système scolaire ou de recherche d'un logement adéquat (Baker et al., 2010; Lai et Ishiyama, 2004). Plus généralement, des études menées en Australie, au Canada, aux États-Unis ou en Nouvelle-Zélande, identifient les mêmes préoccupations des nouveaux arrivants en lien avec un réseau de la santé et des services sociaux complexe (Baker et al., 2010; Lewig et al., 2010; Mortensen et al., 2014; Stewart et al., 2008). 
Afin de faire face aux différentes sources de stress, les parents mobilisent leurs ressources personnelles, mais recourent également à du soutien extérieur, qu'il soit formel ou informel (Gouin et al., 2016; Guralnick et al., 2008). Parmi les dix catégories de stratégie d'aide formelle examinées avec les parents immigrants, certaines s'avèrent davantage utilisées et appréciées que d'autres. Par exemple, le soutien financier, obtenu par l'ensemble des participants, est essentiel pour assumer les coûts liés à la situation de handicap de l'enfant. Quelques parents interrogés jugent cette aide insuffisante et doivent recourir à des organisations caritatives afin de payer leurs dépenses. D'autres études arrivent à des conclusions similaires, illustrant notamment des contraintes pour le remboursement des frais médicaux (Baker et al., 2010; Khanlou et al., 2015). L'accès au marché du travail peut constituer un moyen d'augmenter la capacité financière. Néanmoins, il demande l'organisation de la garde de l'enfant ayant des besoins particuliers. Des obstacles semblables se retrouvent dans d'autres écrits scientifiques (Khanlou et al., 2014; Stewart et al., 2008). Malgré son caractère essentiel, le soutien financier constitue souvent une source de malaise lorsqu'il s'agit de le solliciter. Selon des auteurs (Baker et al., 2010; Canary, 2008), cette réticence à mobiliser du soutien extérieur s'applique à plusieurs catégories d'aide, financières ou non.

Parmi les besoins les plus cités par les parents de cette étude et dans deux autres recherches (Khanlou et al., 2014; Lai et Ishiyama, 2004), le manque d'information semble persister dans le temps. Sachant que la condition de l'enfant évolue lorsqu'il vieillit, les parents doivent sans cesse s'adapter et rechercher de nouvelles informations. Les obstacles pour obtenir des renseignements aux moments opportuns posent des défis communicationnels autant pour les parents que pour les intervenants. À ce sujet, plusieurs auteurs proposent le 
recours à des approches culturellement sensibles (Côté, 2013; Fellin, Desmarais et Lindsay, 2015; King, Desmarais, Lindsay, Piérart et Tétreault, 2015). Elles mettent en évidence l'existence d'une diversité de perceptions de la situation de handicap, d'expressions de ses émotions et de l'importance de l'autonomie, pour ne citer que ces points. Être conscient de sa propre vision du monde et prendre le temps d'explorer et de valider avec les parents immigrants leurs représentations constituent les composantes essentielles d'une bonne communication interculturelle.

En outre, suivant le niveau de connaissances en matière d'organisation sociosanitaire du pays d'accueil, la capacité des parents d'agir seuls semble varier. Grâce à du soutien lors de prises de décisions, les travailleurs sociaux ou les intervenants issus des associations contribuent à favoriser la collaboration des parents lors des prises de décisions. Ce constat, mentionné par les participants, se retrouve dans les écrits scientifiques, mettant l'accent sur les bénéfices liés à cet accompagnement (Canary, 2008; Mortensen et al., 2014). Néanmoins, la diversité des perceptions du soutien peut limiter l'efficience de l'aide apportée, selon les propos recueillis. De même, les méthodes éducatives des parents influencent la manière dont ils considèrent les services de soutien. Plusieurs recherches confirment cette observation (Baker et al., 2010; Lai et Ishiyama, 2004). Par exemple, les multiples interventions en réadaptation peuvent être interprétées comme un frein au parcours scolaire de l'enfant (Baker et al., 2010).

Si certains parents se confient à leur famille (même à distance), ils bénéficient aussi de l'écoute des travailleurs sociaux ou d'intervenants issus des associations. De surcroît, des échanges peuvent avoir lieu avec d'autres parents, lors de réunions (groupes de soutien) ou sur Internet (forums de discussion). Malgré ces différentes ressources, quelques participants 
préfèrent l'intervention d'un psychologue, même si l'accès à ce service semble plus compliqué, comme le confirment Jennings et ses collaboratrices (2014). Moins utilisés et moins connus, les services d'aide pour les loisirs (sport et activités sociales) et pour le transport présentent un intérêt variable selon l'âge de l'enfant, d'après les discours recueillis.

Pour les participants rencontrés, les services de répit sont peu utilisés et le gardiennage est plutôt requis à domicile. Des divergences culturelles entre parents et personnes offrant ces services complexifient parfois la recherche de gardiennes. Quant à l'assistance au quotidien (tâches ménagères, soins à l'enfant), il s'agit du soutien le moins utilisé, soit parce que les ressources informelles sont priorisées, soit parce que les services obtenus ne correspondent pas aux attentes des participants. Ce fait est également mis en évidence par Canary (2008). En effet, l'auteure a observé des situations de stigmatisation de la part de la communauté envers les parents lorsque ces derniers envisageaient de recourir à du soutien formel.

L'analyse de ces résultats permet de faire émerger un modèle explicatif original, inspiré des travaux de McCubbin et Paterson (1983), ainsi que de Tétreault et ses collaborateurs (2014). Il résume le contexte des parents immigrants d'un enfant en situation de handicap en termes d'attentes, de besoins et de recours au soutien formel (Figure 1).

\section{Présentation d'un modèle explicatif}

À la lecture de la figure 1, les situations stressantes que les parents rencontrent (a, aA) et la perception qu'ils en ont $(\mathrm{c}, \mathrm{cC})$ ont été identifiées. De plus, les ressources extérieures (services de soutien informel) (b, bB), utilisées pour faire face au cumul de stress, ont été 
déterminées. Par ailleurs, la satisfaction des parents envers l'aide formelle et leurs besoins non comblés ont été mis en évidence, soulignant les risques de crises ( $\mathrm{x}, \mathrm{xX})$.

Le modèle théorique proposé se base sur des mécanismes avérés, ainsi que sur des écrits scientifiques dont les conclusions diffèrent peu. Cette étude amène une nouvelle compréhension des attentes et des besoins des parents immigrants, et de leurs ressources extérieures (services de soutien formel). Elle met également en évidence l'importance de tenir compte des événements qui se sont déroulés dans le pays d'origine. Il importe alors d'échanger ces personnes au sujet de leur parcours migratoire et de leur situation actuelle. En se basant sur les composantes du modèle présenté, les intervenants (santé, social, éducation) peuvent explorer et valider avec les parents immigrants les différentes sources de stress qu'ils rencontrent. Par exemple, les objectifs et les conditions d'immigration, le statut légal à l'arrivée dans le pays d'accueil, ou encore le moment de la prise de conscience des difficultés de l'enfant peuvent constituer des renseignements utiles à la compréhension du contexte parental. De même, les conséquences de la situation de handicap de l'enfant et de son interprétation doivent être abordées avec les parents. En effet, leur adaptation au pays d'accueil, en plus des soins à l'enfant, entraîne parfois d'autres tensions qui peuvent modifier leurs priorités, leurs attitudes et leurs comportements. C'est pourquoi une approche holistique doit être adoptée par les intervenants. À partir des ressources disponibles, le soutien proposé pourra ainsi être adapté. Cette démarche devrait se poursuivre au fil du temps, par une mise à jour régulièrement de l'ensemble des informations transmises par les parents immigrants. 


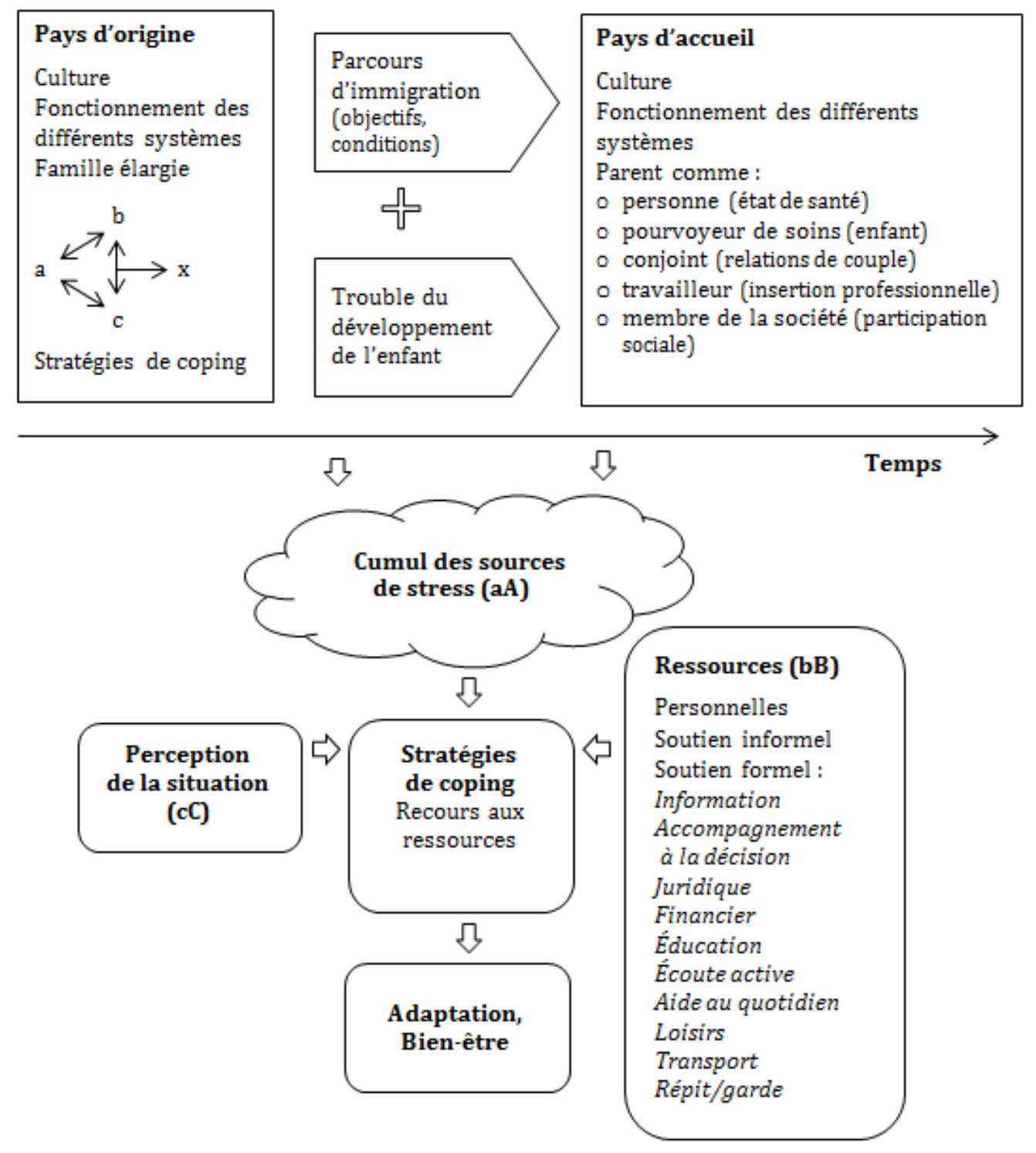

Note : Inspiré de McCubbin et Patterson (1983), et de Tétreault et al. (2014).

Figure 1. Modèle explicatif du recours au soutien par les parents immigrants.

Bien que novatrice, la présente étude comporte certaines limites. D’abord, la taille de l'échantillon s'avère restreinte. Les difficultés de recrutement expliquent le nombre de participants. Néanmoins, les constats ressortis des propos des parents concordent avec les résultats des recherches dans le domaine. Il faut également souligner que les critères de recrutement choisis (avoir reçu des services en lien avec les difficultés de l'enfant et pouvoir s'exprimer en français) n'ont donné accès qu'aux personnes les plus aptes non 
seulement à participer aux entretiens, mais aussi à faire face aux barrières identifiées dans cette recherche. Avec l'aide d'interprètes, il aurait été possible de s'entretenir avec des parents moins outillés qui nécessiteraient un meilleur accompagnement. De surcroît, les propos recueillis doivent être replacés dans le contexte particulier du réseau de la santé et des services sociaux du Québec. Enfin, la complexité des parcours migratoires ainsi que la diversité des atteintes des enfants tendent à privilégier une approche adaptée à chaque famille, leurs caractéristiques et leur cheminement les rendant uniques.

Dans des recherches futures, l'augmentation du nombre de participants permettrait des comparaisons selon différentes dimensions telles que la situation familiale, la date d'arrivée dans le pays d'accueil, le lieu d'origine ou encore le niveau de formation. De plus, l'élargissement des critères de sélection des personnes immigrantes (ne parlant pas la langue du pays d'accueil, par exemple) pourrait contribuer à une meilleure compréhension des défis rencontrés.

\section{Conclusion}

Selon leur parcours migratoire (événements dans le pays d'origine, type d'immigration), les parents d'un enfant en situation de handicap sont susceptibles de vivre davantage d'expériences stressantes que ceux nés au Canada. Le modèle théorique original proposé dans cette étude illustre les multiples sources de difficultés identifiées par les participants en matière de compréhension de l'organisation sociosanitaire du pays d'accueil, d'insertion professionnelle, d'adaptations conjugales et d'isolement social. Néanmoins, les parents rencontrés restent très centrés sur leur enfant, quel que soit son âge. Parmi les ressources extérieures qui leur permettent de faire face, les services de soutien formel s'avèrent parfois 
insuffisants. Par exemple, le manque d'information sur les services actuels, la condition de l'enfant et les perspectives d'avenir ressortent des propos recueillis. En outre, l'aide financière, l'accompagnement administratif et éducatif ainsi que l'écoute active correspondent à des besoins mentionnés comme primordiaux pour les parents rencontrés. Par ailleurs, les services de soutien formel qu'ils reçoivent ne correspondent pas toujours à leurs attentes. La diversité des perceptions de l'éducation des enfants, du soutien et de la réadaptation peut créer des incompréhensions. En documentant les sources de stress et les ressources disponibles directement avec les parents immigrants, les intervenants (santé, social, éducation) contribueraient à optimiser le soutien parental, nécessaire pour accompagner l'enfant en situation de handicap.

\section{Références}

Abbott, D. et Jessiman, P. (2015). "It's afforded us a huge flexibility": The impact of "Disabled Children's Access to Childcare pilots" on families with a disabled child in England. Community, Work and Family, 17(4), 456-466.

Almasri, N. A., O'Neil, M. et Palisano, R. J. (2014). Predictors of needs for families of children with cerebral palsy. Disability and Rehabilitation, 36(3), 210-219.

Alsem, M. W., Siebes, R. C., Gorter, J. W., Jongmans, M. J., Nijhuis, B. G. J. et Ketelaar, M. (2014). Assessment of family needs in children with physical disabilities: Development of a family needs inventory. Child: Care, Health and Development, 40(4), 498-506.

Baker, D. L., Miller, E., Dang, M. T., Yaangh, C.-S. et Hansen, R. L. (2010). Developing culturally responsive approaches with Southeast Asian American families experiencing developmental disabilities. Pediatrics, 126(Suppl. 3), S146-S150.

Ben-Cheikh, I. et Rousseau, C. (2013). Autisme et soutien social dans des familles d'immigration récente : l'expérience de parents originaires du Maghreb. Santé Mentale au Québec, 38(1), 189-205. 
Bourke-Taylor, H., Cotter, C. et Stephan, R. (2014). Young children with cerebral palsy: Families serf-reported equipment needs and out-of-pocket expenditure. Child: Care, Health and Development, 40(5), 654-662.

Canary, H. E. (2008). Creating supportive connections: A decade of research on support for families of children with disabilities. Health Communication, 23(5), 413-426.

Côté, D. (2013). Intercultural communication in health care: Challenges and solutions in work rehabilitation practices and training: A comprehensive review. Disability and Rehabilitation, 35(2), 153-163.

Daudji, A., Eby, S., Foo, T., Ladak, F., Sinclair, C., Landry, M. D., Moody, K. et Gibson, B. E. (2011). Perceptions of disability among South Asian immigrant mothers of children with disabilities in Canada: Implications for rehabilitation service delivery. Disability and Rehabilitation, 33(6), 511-521.

Fellin, M., Desmarais, C. et Lindsay, S. (2015). An examination of clinicians' experiences of collaborative culturally competent service delivery to immigrant families raising a child with a physical disability. Disability and Rehabilitation, 37(21), 1961-1969.

Gouin, J.-P., da Estrela, C., Desmarais, K, et Barker, E. T. (2016). The impact of formal and informal support on health in the context of caregiving stress. Family Relations: Interdisciplinary Journal of Applied Family Studies, 65(1), 191-206.

Guralnick, M. J., Hammond, M. A., Neville, B., et Connor, R. T. (2008). The relationship between sources and functions of social support and dimensions of child- and parentrelated stress. Journal of Intellectual Disability Research, 52(12), 1138-1154.

Hall, H. R. et Graff, J. C. (2011). The relationships among adaptive behaviors of children with autism, family support, parenting stress, and coping. Issues in Comprehensive Pediatric Nursing, 34(1), 4-25.

Harry, B. (2008). Collaboration with culturally and linguistically diverse families: Ideal versus reality. Council for Exceptional Children, 74(3), 372-388.

Hodgetts, S., Zwaigenbaum, L. et Nicholas, D. (2015). Profile and predictors of service needs for families of children with autism spectrum disorders. Autism, 19(6), 673-683.

Imbert, G. (2010). L'entretien semi-directif: à la frontière de la santé publique et de 1'anthropologie. Recherche en Soins Infirmiers, 102(3), 23-34.

Jegatheesan, B., Miller, P. J. et Fowler, S. A. (2010). Autism from a religious perspective: A study of parental beliefs in South Asian Muslim immigrant families. Focus on Autism and other Developmental Disabilities, 25(2), 98-109.

Jennings, S., Khanlou, N. et Su, C. (2014). Public health policy and social support for immigrant mothers raising disabled children in Canada. Disability and Society, 29(10), 1645-1657. 
Khanlou, N., Haque, N., Sheehan, S. et Jones, G. (2014). "It is an issue of not knowing where to go": Service providers' perspectives on challenges in accessing social support and services by immigrant mothers of children with disabilities. Journal of Immigrant and Minority Health, 17(6), 1840-1847.

Khanlou, N., Mustafa, N., Vazquez, L. M., Haque, N. et Yoshida, K. (2015). Stressors and barriers to services for immigrant fathers raising children with developmental disabilities. International Journal of Mental Health and Addiction, 13(6), 659-674.

King, G., Desmarais, C., Lindsay, S., Piérart, G. et Tétreault, S. (2015). The roles of effective communication and client engagement in delivering culturally sensitive care to immigrant parents of children with disabilities. Disability and Rehabilitation, 37(15), 1372-1381.

King, G., Esses, V. M. et Solomon, N. (2013). Immigrant and refugee families raising children with disabling conditions: A review of the international literature on service access, service utilization, and service care experiences. Dans E. L. Grigorenko (Ed.), Handbook of US immigration and education (p. 179-206). New-York, NY: Springer.

Lai, Y. et Ishiyama, F. I. (2004). Involvement of immigrant Chinese Canadian mothers of children with disabilities. Council for Exceptional Children, 71(1), 97-108.

Lawton, K. E., Gerdes, A. C., Haack, L. M. et Schneider, B. (2014). Acculturation, cultural values, and Latino parental beliefs about the etiology of ADHD. Administration and Policy in Mental Health and Mental Health Services Research, 41(2), 189-204.

Lewig, K., Arney, F. et Salveron, M. (2010). Challenges to parenting in a new culture: Implications for child and family welfare. Evaluation and Program Planning, 33(3), 324332.

Lin, M.-C, Macmillan, M. et Brown, N. (2010). The formal support experiences of mothers of adolescents with intellectual disabilities in Edinburgh, UK: A longitudinal qualitative design. Journal of Nursing Research, 18(1), 34-42.

McCubbin, H. I. et Patterson, J. M. (1983). The family stress process: The double ABCX model of adjustment and adaptation. Dans H. I. McCubbin, M. B. Sussman et J. M. Patterson (Eds.), Social stress and the family: Advances and developments in family stress theory and research (p. 7-37). New York, NY: The Haworth Press.

Miles, M. B. et Huberman, A. M. (2003). Analyse des données qualitatives ( $2^{\mathrm{e}}$ éd.). Bruxelles, Belgique : De Boeck Université.

Mortensen, A., Latimer, S. et Yusuf, I. (2014). Cultural case workers in child disability services: An evidence-based model of cultural responsiveness for refugee families. Kōtuitui: New Zealand Journal of Social Sciences Online, 9(2), 50-59. 
Neely-Barnes, S. L. et Dia, D. A. (2008). Families of children with disabilities: A review of literature and recommendations for interventions. Journal of Early and Intensive Behavior Intervention, 5(3), 93-107.

Paillé, P. et Mucchielli, A. (2012). L'analyse qualitative en sciences humaines et sociales ( $3^{\mathrm{e}}$ éd.). Paris, France : Armand Colin.

Pondé, M. P. et Rousseau, C. (2013). Immigrant children with autism spectrum disorder: The relationship between the perspective of the professionals and the parents' point of view. Journal of the Canadian Academy of Child and Adolescent Psychiatry, 22(2), 131138.

Povenmire-Kirk, T. C., Lindstrom, L. et Bullis, M. (2010). De escuela a la vida adulta/From school to adult life: Transition needs for Latino youth with disabilities and their families. Career Development for Exceptional Individuals, 33(1), 41-51.

Pozo, P., Sarriá, E. et Brioso, A. (2014). Family quality of life and psychological wellbeing in parents of children with autism spectrum disorders: A double ABCX model. Journal of Intellectual Disability Research, 58(5), 442-458.

Riggs, E., Davis, E., Gibbs, L., Block, K., Szwarc, J., Casey, S., Duell-Piening, P. et Waters, E. (2012). Accessing maternal and child health services in Melbourne, Australia: Reflections from refugee families and service providers. BMC Health Services Research, 12, 1-16. Repéré sur le site de la revue : https://bmchealthservres.biomedcentral.com/articles/10.1186/1472-6963-12-117

Stewart, M., Anderson, J., Morton, B., Mwakarimba, E., Neufeld, A., Simich, L. et Spitzer, D. (2008). Multicultural meanings of social support among immigrants and refugees. International Migration, 46(3), 123-159.

Tétreault, S., Blais-Michaud, S., Marier-Deschênes, P., Beaupré, P., Gascon, H., Boucher, N. et Carrière, M. (2014). How to support families of children with disabilities? An exploratory study of social support services. Child and Family Social Work, 19(3), 272281.

Weiss, J. A., Tint, A., Paquette-Smith, M. et Lunsky, Y. (2016). Perceived self-efficacy in parents of adolescents and adults with autism spectrum disorder. Autism, 20(4), 425-434.

Xue, J., Ooh, J. et Magiati, I. (2014). Family functioning in Asian families raising children with autism spectrum disorders: The role of capabilities and positive meanings. Journal of Intellectual Disability Research, 58(5), 406-420.

Zuckerman, K. E., Sinche, B., Cobian, M., Cervantes, M., Mejia, A., Becker, T. et Nicolaidis, C. (2014). Conceptualization of autism in the Latino community and its relationship with early diagnosis. Journal of Developmental and Behavioral Pediatrics, $35(8), 522-532$. 